\title{
Cedecea lapagei
}

National Cancer Institute

\section{Source}

National Cancer Institute. Cedecea lapagei. NCI Thesaurus. Code C86247.

A species of facultatively anaerobic, Gram-negative, bacilli assigned to the phylum

Proteobacteria. This species is motile, non-spore forming, catalase positive, oxidase negative, lipase positive, indole negative, ornithine decarboxylase negative, does not ferment sucrose, reduces nitrate, does not produce hydrogen sulfide, and hydrolyzes esculin. C. lapagei is pathogenic especially in immunocompromised patients. 